\title{
Validation of the Dutch Version of the M.D. Anderson Dysphagia Inventory for Neurogenic Patients
}

\author{
Eveline E. Samuels ${ }^{a, b}$ Michel van Hooren ${ }^{c, d}$ Laura W.J. Baijens ${ }^{c, e}$ \\ Anne-Sophie Beeckman ${ }^{b, f}$ Valéria Lima Passos ${ }^{g, h}$ Walmari Pilz ${ }^{c, d}$ \\ aDepartment of Speech and Language Pathology, Maastricht University Medical Center, Maastricht, The

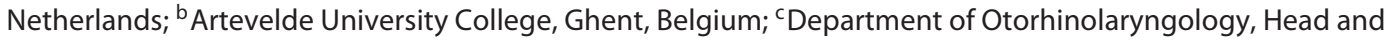 \\ Neck Surgery, Maastricht University Medical Center, Maastricht, The Netherlands; ${ }^{d}$ School for Mental Health

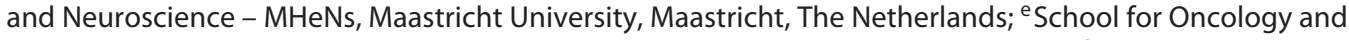 \\ Developmental Biology - GROW, Maastricht University, Maastricht, The Netherlands; ${ }^{f}$ Department of Speech and \\ Language Pathology, Maria Middelares Hospital, Ghent, Belgium; 9 Department of Methodology and Statistics, \\ Maastricht University, Maastricht, The Netherlands; ${ }^{\mathrm{h}}$ Care and Public Health Research Institute - CAPHRI, Maastricht \\ University, Maastricht, The Netherlands
}

\section{Keywords}

M.D. Anderson Dysphagia Inventory · Dysphagia . Quality-of-life questionnaire - Validity · Deglutition . Deglutition disorders

\begin{abstract}
Background/Aim: The aim of this study was to validate the Dutch-language version of the M.D. Anderson Dysphagia Inventory (MDADI) for patients with neurogenic oropharyngeal dysphagia (OD). Methods: One hundred and seventyeight patients with neurogenic OD and 92 healthy control subjects completed the MDADI and the Dutch version of the Swallowing Quality-of-Life Questionnaire (SWAL-QOL-NL). Exclusion criteria were: suffering from a concurrent headand-neck oncological disease, scoring below 23 on a Mini Mental State Examination, being older than 85 years, and being illiterate or blind. None of the patients was in a palliative state of disease. Floor and ceiling effects, known-groups validity, internal consistency, construct validity, and criterion
\end{abstract}

validity were assessed. Results: The MDADI total score showed no floor or ceiling effects for the patient group. Known-groups validity was confirmed by group differences in score distributions between patients and healthy control subjects. The internal consistency showed Cronbach's $a$-values ranging from 0.77 to 0.92 . Correlations between the MDADI subscales and SWAL-QOL-NL domains were moderate to strong: $0.71,0.70$, and 0.62 (convergent construct validity). Correlations between the MDADI scores and the SWAL-QOL-NL domains general burden, food selection, eating duration, communication, mental health, social functioning, and frequency of symptoms were moderate to strong, ranging from 0.41 to 0.75 . Weak correlations $(<0.4)$ were found between the MDADI scores and the SWAL-QOLNL domains eating desire, sleep, and fatigue. Conclusion: The results of this study show that the Dutch translation of the MDADI is a psychometrically validated and suitable dysphagia-specific quality-of-life questionnaire for patients with neurogenic OD.

(C) 2019 The Author(s)

Published by S. Karger AG, Basel
(C) 2019 The Author(s)

Published by S. Karger AG, Basel

This article is licensed under the Creative Commons AttributionNonCommercial-NoDerivatives 4.0 International License (CC BYNC-ND) (http://www.karger.com/Services/OpenAccessLicense) Usage and distribution for commercial purposes as well as any distribution of modified material requires written permission.
Laura W.J. Baijens

Department of Otorhinolaryngology, Head and Neck Surgery

Maastricht University Medical Center

PO Box 5800, NL-6202 AZ Maastricht (The Netherlands)

E-Mail laura.baijens@mumc.nl 


\section{Introduction}

Oropharyngeal dysphagia (OD) is a common finding in patients suffering from neurogenic disorders. Swallowing impairment can be acute or chronic in nature. Acute OD is observed in patients after a stroke, head injury, neurosurgical intervention, or in patients with GuillainBarré syndrome, for instance. Patients who do not recover after stroke can develop chronic OD. Degenerative OD is seen in patients with progressive neurological diseases such as Parkinson's disease, amyotrophic lateral sclerosis, myasthenia gravis, Huntington's disease, myotonic dystrophy type 1, and multiple sclerosis [1]. All 4 stages of the swallowing process can be affected: the preparatory, the oral, the pharyngeal, and the esophageal stage. Generally speaking, as neurological disease severity increases, so does OD [1]. The prevalence of OD in neurogenic patients ranges from 3 to $50 \%$ in stroke patients $[2,3]$ and to almost $100 \%$ in patients suffering from Huntington's disease [4]. OD can cause weight loss, malnutrition, social isolation, aspiration pneumonia, and decreased healthrelated quality of life (QoL) [5].

A study performed in 5 European countries showed that swallowing disorders have a major impact on the health-related QoL of dysphagic patients [6]. Some studies reported that dysphagia-specific QoL was poorly correlated with, among other things, the severity of OD as measured using fiberoptic endoscopic evaluation of swallowing (FEES) and videofluoroscopy of swallowing (VFS) [7-9]. Therefore, used alongside these instrumental assessments, dysphagia-specific QoL questionnaires add value by providing insight into patients' perception of OD, which can be taken into account in the treatment plan.

Nowadays several validated dysphagia-specific QoL questionnaires are available in the Dutch language: the Swallowing Quality-of-Life Questionnaire (SWAL-QOL, 44 items) [10-12], the Deglutition Handicap Index (30 items) $[13,14]$, the Dysphagia Handicap Index (25 items) $[15,16]$, and the M.D. Anderson Dysphagia Inventory (MDADI, 20 items) $[14,17]$. The multidisciplinary dysphagia clinics in the Netherlands are mainly visited by patients with OD of head-and-neck oncological or neurological origin [18]. A smaller proportion of patients have a heterogeneous etiology of OD: cervical osteophyte formation, frail elderly age, Zenker diverticulum, cervical hernia surgery, congenital mental impairment, syndromic diseases, and so on. As such, these dysphagia clinics offer a suitable setting for deploying a questionnaire that could be applied to all OD patients irrespective of the un- derlying etiology. Among all questionnaires available in the Dutch language, the MDADI seemed the most suitable choice, because it allows global judgment of the dysphagia-specific QoL and it is easy to implement in a busy daily otorhinolaryngological outpatient clinic for OD. Other questionnaires such as the SWAL-QOL-NL and the Deglutition Handicap Index are longer than the MDADI and take more time to complete. The aim of this study was to validate the Dutch version of the MDADI for patients with neurogenic OD.

\section{Methods}

\section{Subjects}

Patients with neurogenic disorders and OD were recruited from an otorhinolaryngological outpatient clinic for OD at a tertiary university referral hospital between January 2009 and October 2016. Data were also collected from healthy control subjects in the local community who had no swallowing complaints and whose overall health was good. Exclusion criteria were: a Mini Mental State Examination score below 23 points [19], blindness, illiteracy, a history of head and neck cancer (HNC), and age below 18 or above 85 years. Informed consent was obtained from all patients for clinical purposes, and the study protocol was approved by the medical Ethics Committee according to the non-WMO obligatory Medical Research Involving Human Subjects Act.

\section{M.D. Anderson Dysphagia Index}

The MDADI is a self-administered, psychometrically validated dysphagia-specific questionnaire for HNC patients that is designed to assess the impact of OD on health-related QoL [17]. Like the original English version, the validated Dutch translation of the MDADI consists of 20 items pooled in 4 subscales: the global scale ( 1 item); the functional scale (5 items); the physical scale ( 8 items); and the emotional scale ( 6 items) [14]. The global assessment question (MDADI-G) evaluates the effect of swallowing disability on overall QoL. The functional scale (MDADI-F) illustrates the impact of OD on daily activities. The physical scale (MDADI-P) measures the patient's self-perception of the physical impact of OD. The emotional scale (MDADI-E) represents the patient's affective response to the swallowing disorder in terms of embarrassment, self-esteem, and self-consciousness. All items are scored on a 5 -point scale (1-5), where "1" corresponds to "total agreement" and " 5 " to "total disagreement." In the original version of the MDADI, all but 2 items were scored such that higher scores indicated higher functioning [17]. In the Dutch translation, it was decided to use a uniform scoring method [14]. Thus, by adjusting the scoring of these 2 items, low scores came to indicate low functioning and high scores high functioning. Responses on all domains were summed to calculate the total score (MDADI-T). The maximum score is 100 , indicating high functioning, and the minimum score is 20 , indicating poor functioning.

Dutch Version of the SWAL-QOL

The SWAL-QOL questionnaire was designed to evaluate the impact of OD on health-related QoL in dysphagic patients. It consists of 44 items divided among 11 domains: general burden (2 
Table 1. Definition of statistical terms

\begin{tabular}{|c|c|}
\hline Term & Definition \\
\hline Floor and ceiling effect & The number of respondents that achieved the lowest or highest possible score \\
\hline Internal consistency & The extent to which items in a (sub)test measure the same concept \\
\hline Construct validity & $\begin{array}{l}\text { The extent to which scores of a questionnaire correlate with other measurements concerning the } \\
\text { hypotheses put forward }\end{array}$ \\
\hline Convergent construct validity & $\begin{array}{l}\text { The extent of correlation between scores on } 2 \text { different questionnaires that aim to measure the } \\
\text { same concept (the closer to } 1 \text {, the higher the correlation) }\end{array}$ \\
\hline Discriminant construct validity & $\begin{array}{l}\text { The extent of correlation between scores on } 2 \text { different questionnaires that do not aim to measure } \\
\text { the same concept (the closer to } 0 \text {, the higher the correlation) }\end{array}$ \\
\hline Criterion validity & The extent to which scores of a questionnaire correlate with the gold standard measurement \\
\hline
\end{tabular}

items); food selection ( 2 items); eating duration (2 items); eating desire (3 items); fear of eating ( 4 items); sleep (2 items); fatigue (3 items); communication ( 2 items); mental health (5 items); social functioning (5 items); and frequency of symptoms ( 14 items). Each item is scored on a 5-point scale: the higher the score, the better the swallow-related QoL. Completion of the questionnaire takes 15-30 min. The Dutch version of the SWAL-QOL (SWAL-QOL$\mathrm{NL}$ ) is considered the gold standard for determining dysphagiaspecific QoL in patients with OD $[11,12,14,16,20,21]$.

Two studies translated the SWAL-QOL questionnaire into the Dutch language and validated it for a mixed population of dysphagic patients: SWAL-QOL-NL and DSWAL-QOL $[11,12]$. In the current study, the SWAL-QOL-NL was used as a gold standard for the validation of the MDADI. In the validation study of the SWAL-QOLNL, 2 domains - "eating desire" and "communication" - did not reach sufficient internal validity (Cronbach's $\alpha=0.67$ and 0.6 , respectively) and were removed from the final questionnaire. As the internal consistency is greatly dependent on the underlying population under scrutiny, it was decided to use the 44 items of the SWAL-QOL version, which was used to validate the original SWAL-QOL-NL, in the present study too in order to establish and present the psychometric properties of the questionnaire for the current target sample.

\section{Statistical Analysis}

Floor and ceiling effects of the MDADI-T and subscales were defined as evident effects when $15 \%$ or more of the patients obtained the lowest or highest possible scores and were recorded as proportions (\%) of the extreme scores (20-100) [22]. Given the skewness of the data, the 2 groups (patients and healthy control subjects) were compared for known-groups validity using the Mann-Whitney $U$ test. The internal consistency of the MDADI and the SWAL-QOL-NL was determined using Cronbach's $\alpha$-value. The lowest acceptable level of internal consistency was set at $\alpha<0.70$ [22]. Convergent construct and criterion validities were assessed using Spearman's correlation, as were all validities. Cor- relations between 0.00 and 0.19 were considered very weak, between 0.20 and 0.39 weak, between 0.40 and 0.59 moderate, between 0.60 and 0.79 strong, and between 0.80 and 1.0 very strong [23]. Regarding convergent construct validity, it was hypothesized that the MDADI-F would show a strong correlation with the SWAL-QOL-NL domain "social functioning," the MDADI-P with the SWAL-QOL-NL domain "frequency of symptoms," and the MDADI-E with the SWAL-QOL-NL domain "mental health." Regarding discriminant construct validity, it was hypothesized that the SWAL-QOL-NL domains "sleep" and "fatigue" would show a weak correlation with all MDADI subscales. Statistical analysis was performed using SPSS 24 (IBM, SPSS Inc., Chicago, IL, USA). Given the multitude of statistical tests conducted, type I error inflation was controlled for via step-down Bonferroni (Holm) correction. A statistical effect was determined if the $p$ value was $<0.05$ following adjustment for multiple testing. Table 1 provides the reader with a short definition of some of the statistical terms used here.

\section{Results}

\section{Characteristics of the Population}

The patient group comprised 178 persons $(n=113$ men, $64 \%$ ) with a mean age of 59 years, ranging from 21 to 82 (SD 15). Their diagnoses were Parkinson's disease $(n=94,52.8 \%)$, myotonic dystrophy type $1(n=60,33.7 \%)$, and other neurogenic diseases such as stroke, multiple sclerosis, and amyotrophic lateral sclerosis $(n=24,13.5 \%)$. All included patients completed the Dutch version of the MDADI and the SWAL-QOL-NL. Ninety-two healthy control subjects ( 45 men) were recruited from the local community, and their mean age was 47 years, ranging
Samuels et al. 
Table 2. Group differences in MDADI scores

\begin{tabular}{|c|c|c|c|c|c|}
\hline \multirow{2}{*}{$\begin{array}{l}\text { MDADI } \\
\text { subscales }\end{array}$} & \multirow{2}{*}{$\begin{array}{l}\text { Healthy control group } \\
\text { ( } n=55) \text { median } \\
\text { (25th-75th percentiles) }\end{array}$} & \multicolumn{3}{|c|}{ Patient group $(n=178)$} & \multirow[t]{2}{*}{$p$ value } \\
\hline & & $\begin{array}{l}\text { median } \\
\text { (25th-75th } \\
\text { percentiles) }\end{array}$ & $\begin{array}{l}\text { ceiling } \\
\text { effect, \% }\end{array}$ & $\begin{array}{l}\text { frequency } \\
\text { distribution }\end{array}$ & \\
\hline Global & $5(5-5)$ & $4(2-5)$ & 30.3 & & $<0.001$ \\
\hline Functional & $25(25-25)$ & $21(19-35)$ & 24.3 & & $<0.001$ \\
\hline Physical & $40(40-40)$ & $29(23-25)$ & 9.0 & & $<0.001$ \\
\hline Emotional & $30(29-30)$ & $22(18-25)$ & 10.1 & & $<0.001$ \\
\hline Total & $100(99-100)$ & $75(65-85)$ & 8.4 & & $<0.001$ \\
\hline
\end{tabular}

MDADI, M.D. Anderson Dysphagia Inventory; $p$ value assessed by the Mann-Whitney U test.

from 20 to 82 (SD 15). Fifty-five healthy subjects completed the MDADI and 37 completed the SWAL-QOLNL. All subjects were native speakers of Dutch.

\section{Floor and Ceiling Effects}

Tables 2 and 3 present the descriptive statistics for the healthy control subjects and the patient group. No floor effect was detected for any of the MDADI subscales in the patient group. However, a ceiling effect was found for the MDADI-G and the MDADI-F subscales. In the healthy control group, a ceiling effect was found for all MDADI subscales and the MDADI-T.

\section{Known-Groups Validity}

The median MDADI scores (total and subscales) were significantly lower for the patient group than for the healthy control group (Table 2). The median (25th-75th percentiles) of the MDADI-T scores for patients was 75 (65-85); for the healthy control group it was 100 (99-100; $p<0.001)$. As such, the MDADI questionnaire was able to distinguish between those with and those without OD, as evidenced by statistically significant group differences for all MDADI subscales and MDADI-T (Table 2).

\section{Internal Consistency}

Cronbach's a ranged from 0.77 to 0.92 , showing a good internal consistency; that is, the items on each subscale of the MDADI questionnaire measure the same general construct (Table 4). The internal consistency of the domains of the SWAL-QOL-NL appeared to be good too, with values ranging from $\alpha=0.76$ to 0.90 . Only the domain "frequency of symptoms" showed a weaker correlation between the items $(\alpha=0.62)$.

\section{Construct Validity}

Moderate to strong correlations were found between the MDADI-F subscale and the domain "social functioning" of the SWAL-QOL-NL, between the MDADI$P$ subscale and the SWAL-QOL-NL domain "frequency of symptoms," and between the MDADI-E subscale and the SWAL-QOL-NL domain "mental health" (Table 5). The correlation coefficients between the domains "sleep" and "fatigue" of the SWAL-QOL-NL and the different subscales of the MDADI were either low or very low (Table 5). These results support the hypothesis about convergent and discriminant construct validity. 
Table 3. Median and percentiles of SWAL-QOL-NL scores for the healthy control and patient groups

\begin{tabular}{lcl}
\hline $\begin{array}{l}\text { SWAL-QOL-NL } \\
\text { domains }\end{array}$ & $\begin{array}{l}\text { Healthy control } \\
\text { group }(n=37), \\
\text { median }(25 \text { th- } \\
\text { 75th percentiles })\end{array}$ & $\begin{array}{l}\text { Patient group } \\
(n=178), \\
\text { median }(25 \text { th- } \\
75 \text { th percentiles })\end{array}$ \\
\hline General burden & $100(100-100)$ & $63(38-88)$ \\
Food selection & $100(100-100)$ & $75(63-100)$ \\
Eating duration & $100(100-100)$ & $50(25-89)$ \\
Eating desire & $100(92-100)$ & $75(48-100)$ \\
Fear of eating & $100(100-100)$ & $91(75-100)$ \\
Sleep & $88(75-100)$ & $75(38-88)$ \\
Fatigue & $92(79-100)$ & $58(33-75)$ \\
Communication & $100(100-100)$ & $63(38-75)$ \\
Mental health & $100(100-100)$ & $80(60-95)$ \\
Social functioning & $100(100-100)$ & $75(55-100)$ \\
Frequency of symptoms & $88(75-100)$ & $64(95-100)$
\end{tabular}

SWAL-QOL-NL, the Dutch version of the Swallowing Qualityof-Life Questionnaire.

\section{Criterion Validity}

Table 5 shows the correlations between the MDADI subscales and the SWAL-QOL-NL domains in the patient group. All correlations were significant, except for the correlation between the MDADI-F subscale and the SWAL-QOL-NL domain "fatigue."

\section{Discussion}

Instrumental assessment tools such as FEES or VFS provide valuable information about the nature and severity of a swallowing disorder but they do not provide any information on the dimension of how the swallowing impairment affects a patient's life. Dysphagia-specific health-related QoL questionnaires can help the patient formulate his/her perception of the swallowing disorder [9-12]. Moreover, these questionnaires enable clinicians to detect what is important to the patients; the subjective responses can be useful when discussing patients' motivation for therapy. Therapy effects of OD treatment in HNC patients can be further quantified using for example the MDADI or the SWAL-QOL-NL.

Clinical practice has a manifest need for validated, easy-to-use dysphagia-specific questionnaires that can be filled in quickly by patients with neurogenic OD. Such a questionnaire is currently not available in the Dutch language. In the present study the preferred dysphagia-spe-
Table 4. Internal consistency of the MDADI subscales and SWALQOL-NL domains in the patient group

\begin{tabular}{lcc}
\hline Instrument & Items & Cronbach's a \\
\hline MDADI & & \\
$\quad$ Global & 1 & - \\
Functional & 5 & 0.77 \\
Physical & 8 & 0.85 \\
Emotional & 6 & 0.79 \\
Total & 19 & 0.92 \\
SWAL-QOL-NL & & \\
General burden & 2 & 0.90 \\
Food selection & 2 & 0.80 \\
Eating duration & 2 & 0.80 \\
Eating desire & 3 & 0.78 \\
Fear of eating & 4 & 0.80 \\
Sleep & 2 & 0.82 \\
Fatigue & 3 & 0.85 \\
Communication & 2 & 0.76 \\
Mental health & 5 & 0.88 \\
Social functioning & 5 & 0.93 \\
Frequency of symptoms & 14 & 0.62 \\
\hline
\end{tabular}

MDADI, M.D. Anderson Dysphagia Inventory; SWAL-QOLNL, the Dutch version of the Swallowing Quality-of-Life Questionnaire.

cific QoL questionnaire to validate for neurogenic patients in the Dutch language was the MDADI as the MDADI was already validated for Dutch HNC patients and can be distributed for both patient groups during clinical practice at otorhinolaryngological outpatient clinics for OD. The MDADI questionnaire is considerably shorter and therefore more convenient for neurological patients compared to the validated alternatives available in the Dutch language (SWAL-QOL, Deglutition Handicap Index) [13, 14]. The items in the MDADI questionnaire are formulated in such a way that they do not contain any head-andneck cancer-specific elements. This too was an argument for choosing this questionnaire, which had already been officially translated into Dutch. The time required to complete the MDADI questionnaire by neurogenic patients visiting the present outpatient setting was on average 5 min. The original MDADI study by Chen et al. [17] examined psychometric properties of the questionnaire comprising known-groups validity, reliability (internal consistency and test-retest), construct validity, and criterion validity. The results showed that the MDADI is a reliable and valid questionnaire to evaluate the impact of OD on HNC patients' health-related QoL [17]. However, the MDADI was never validated in a group consisting entirely of neu- 
Table 5. Criterion validity: correlations between the MDADI subscales and SWAL-QOL-NL domains in the patient group

\begin{tabular}{|c|c|c|c|c|c|}
\hline \multirow[t]{2}{*}{ SWAL-QOL-NL domains } & \multicolumn{5}{|c|}{ MDADI subscales } \\
\hline & $\begin{array}{l}\text { global scale } \\
p \text { value }\end{array}$ & $\begin{array}{l}\text { functional scale } \\
p \text { value }\end{array}$ & $\begin{array}{l}\text { physical scale } \\
p \text { value }\end{array}$ & $\begin{array}{l}\text { emotional scale } \\
p \text { value }\end{array}$ & $\begin{array}{l}\text { total scale } \\
p \text { value }\end{array}$ \\
\hline General burden & $\begin{array}{r}0.573 \\
\leq 0.001\end{array}$ & $\begin{array}{r}0.488 \\
\leq 0.001\end{array}$ & $\begin{array}{r}0.549 \\
\leq 0.001\end{array}$ & $\begin{array}{r}0.571 \\
\leq 0.001\end{array}$ & $\begin{array}{r}0.590 \\
\leq 0.001\end{array}$ \\
\hline Food selection & $\begin{array}{r}0.523 \\
\leq 0.001\end{array}$ & $\begin{array}{r}0.513 \\
\leq 0.001\end{array}$ & $\begin{array}{r}0.620 \\
\leq 0.001\end{array}$ & $\begin{array}{r}0.577 \\
\leq 0.001\end{array}$ & $\begin{array}{r}0.671 \\
\leq 0.001\end{array}$ \\
\hline Eating duration & $\begin{array}{r}0.484 \\
\leq 0.001 \\
\end{array}$ & $\begin{array}{r}0.422 \\
\leq 0.001 \\
\end{array}$ & $\begin{array}{r}0.598 \\
\leq 0.001 \\
\end{array}$ & $\begin{array}{r}0.519 \\
\leq 0.001 \\
\end{array}$ & $\begin{array}{r}0.563 \\
\leq 0.001 \\
\end{array}$ \\
\hline Eating desire & $\begin{array}{r}0.328 \\
\leq 0.001\end{array}$ & $\begin{array}{r}0.372 \\
\leq 0.001\end{array}$ & $\begin{array}{r}0.375 \\
\leq 0.001\end{array}$ & $\begin{array}{r}0.369 \\
\leq 0.001\end{array}$ & $\begin{array}{r}0.408 \\
\leq 0.001\end{array}$ \\
\hline Fear of eating & $\begin{array}{r}0.381 \\
\leq 0.001 \\
\end{array}$ & $\begin{array}{r}0.333 \\
\leq 0.001\end{array}$ & $\begin{array}{r}0.446 \\
\leq 0.001 \\
\end{array}$ & $\begin{array}{r}0.450 \\
\leq 0.001\end{array}$ & $\begin{array}{r}0.427 \\
\leq 0.001 \\
\end{array}$ \\
\hline Sleep & $\begin{array}{l}0.204 \\
0.018\end{array}$ & $\begin{array}{l}0.182 \\
0.032\end{array}$ & $\begin{array}{l}0.229 \\
0.012\end{array}$ & $\begin{array}{l}0.231 \\
0.012\end{array}$ & $\begin{array}{l}0.252 \\
0.009\end{array}$ \\
\hline Fatigue & $\begin{array}{r}0.284 \\
\leq 0.001\end{array}$ & $\begin{array}{r}0.110 \\
\leq 0.145\end{array}$ & $\begin{array}{l}0.247 \\
0.009\end{array}$ & $\begin{array}{l}0.249 \\
0.009\end{array}$ & $\begin{array}{l}0.232 \\
0.012\end{array}$ \\
\hline Communication & $\begin{array}{r}0.448 \\
\leq 0.001\end{array}$ & $\begin{array}{r}0.458 \\
\leq 0.001\end{array}$ & $\begin{array}{r}0.489 \\
\leq 0.001\end{array}$ & $\begin{array}{r}0.513 \\
\leq 0.001\end{array}$ & $\begin{array}{r}0.522 \\
\leq 0.001\end{array}$ \\
\hline Mental health & $\begin{array}{r}0.614 \\
\leq 0.001\end{array}$ & $\begin{array}{r}0.591 \\
\leq 0.001\end{array}$ & $\begin{array}{r}0.672 \\
\leq 0.001\end{array}$ & $\begin{array}{r}0.701 \\
\leq 0.001\end{array}$ & $\begin{array}{r}0.719 \\
\leq 0.001\end{array}$ \\
\hline Social functioning & $\begin{array}{r}0.653 \\
\leq 0.001\end{array}$ & $\begin{array}{r}0.709 \\
\leq 0.001\end{array}$ & $\begin{array}{r}0.663 \\
\leq 0.001\end{array}$ & $\begin{array}{r}0.736 \\
\leq 0.001\end{array}$ & $\begin{array}{r}0.754 \\
\leq 0.001\end{array}$ \\
\hline Frequency of symptoms & $\begin{array}{r}0.516 \\
\leq 0.001\end{array}$ & $\begin{array}{r}0.451 \\
\leq 0.001\end{array}$ & $\begin{array}{r}0.624 \\
\leq 0.001\end{array}$ & $\begin{array}{r}0.523 \\
\leq 0.001\end{array}$ & $\begin{array}{r}0.626 \\
\leq 0.001\end{array}$ \\
\hline
\end{tabular}

MDADI, M.D. Anderson Dysphagia Inventory; SWAL-QOL-NL, the Dutch version of the Swallowing Quality-of-Life Questionnaire. In each SWAL-QOL-NL domain, the first number indicates Spearman's correlation coefficient.

rological patients. To our knowledge, this is the first study intended to validate the Dutch version of the MDADI questionnaire for patients with neurogenic OD.

Divergent statistical validation techniques were applied across the published MDADI validation studies in different languages (Dutch, Japanese, Portuguese, Italian, Chinese, Swedish, etc.) [14, 24-30]. These studies assessed different sets of psychometric properties such as floor and ceiling effects, known-groups validity, reliability (internal consistency and test-retest reliability), construct validity, criterion validity, and so on. To validate the MDADI questionnaire in patients with $\mathrm{OD}$ of neurological origin in the present study, the following aspects were examined: floor and ceiling effect, known-groups validity, internal consistency, construct validity, and criterion validity. Similar to the study validating the MDADI for HNC patients, there was no floor and/or ceiling effect for the MDADI-T in neurogenic patients in the present study. Analysis of the individual MDADI subscales identified a ceiling effect for MDADI-G and -F. However, when all the subscales were observed in conjunction (MDADI-T), only $8.4 \%$ of the patients achieved high scores (thus a weak indication for a ceiling effect). Furthermore, the results showed that the distributions of the MDADI scores differed significantly between subjects with and without OD (patients vs. healthy control subjects). When comparing the results with those of the Swedish MDADI validation study [24], which is the only 
one that included a group of neurogenic patients, the internal consistency for the MDADI-T score of neurogenic patients was found to be the same as in the present study. For the floor and ceiling effects, similar results were found. The construct validity of the Swedish study could not be compared with that of the present study as the Swedish group had tested the MDADI against the ShortForm 36 and the Hospital Anxiety and Depression Scale.

The criterion validity ranged from acceptable to high. The correlations between the MDADI-T and the SWALQOL-NL domains "sleep" and "fatigue" were weak. The Dutch MDADI validation study by Speyer et al. [14] found a marginally higher correlation coefficient for "sleep" and "fatigue" but their results were similar to the findings in the present study. The reason for these weak correlations can probably be found in the fact that the specific domains "sleep" and "fatigue" of the SWAL-QOL-NL are not represented by similar items in the MDADI questionnaire. The Dutch version of the MDADI is a valid questionnaire that allows clinicians to gain insight into the dysphagiaspecific QoL of dysphagic patients with neurogenic disorders. Implementation of this questionnaire as part of a multidimensional swallowing assessment for neurological patients in daily clinical practice is recommended because it may add clinical value when used alongside instrumental swallowing assessment tools such as FEES and VFS, which visualize other dimensions of the swallowing impairment. Information on the dimension how the patient perceives the impact of OD on his/her health-related QoL, in combination with clinical and instrumental swallowing evaluation, provides the opportunity to offer a patienttailored treatment program based on his/her needs and expectations. Consequently, it might improve patients' compliance to and satisfaction about the treatment.

\section{Limitations and Future Directions}

With regard to reliability, only the internal consistency was studied. In the future, test-retest reliability could be examined by filling in the MDADI questionnaire at 2 different measurement times [31]. Responsiveness of the questionnaire could be examined as well if the MDADI could be filled in at several time points by the same patients with neurogenic OD. That would reveal whether the questionnaire captures changes in the health status, changes in disease progression, and changes due to therapy effects. Finally, the patient population in this study was recruited in one location at an otorhinolaryngological outpatient clinic for OD.

\section{Conclusion}

The results of this study show that the Dutch translation of the MDADI is a psychometrically validated and suitable dysphagia-specific QoL questionnaire for patients with neurogenic OD.

\section{Statement of Ethics}

Informed consent was obtained from all patients for clinical purposes, and the study protocol was approved by the institutional medical Ethics Committee according to the non-WMO obligatory Medical Research Involving Human Subjects Act in the Netherlands (http://www.ccmo.nl/en/non-wmo-research).

\section{Disclosure Statement}

There was no grant support, and the authors declare that they have no conflict of interest.

\section{Author Contributions}

Each author has made substantial contributions to the (1) design and data collection of this original manuscript, or to the acquisition, data analysis, or interpretation of data including statistical analysis for this manuscript, (2) participated in drafting the work or revising it critically for important intellectual content, (3) approved the final version to be published, and (4) agreed to be accountable for all aspects of the work in ensuring that questions related to the accuracy or integrity of any part of the work are appropriately investigated and resolved.

\section{References}

1 De Bodt M, Guns C, D’Hondt M, Vanderwegen J, Van Nuffelen G. Dysfagie, Handboek voor de klinische praktijk. Antwerpen: Garant; 2015.

2 Smithard DG, O'Neill PA, England RE, Park CL, Wyatt R, Martin DF, et al. The natural history of dysphagia following a stroke. Dysphagia. 1997;12(4):188-93.
3 Mann G, Hankey GJ, Cameron D. Swallowing function after stroke: prognosis and prognostic factors at 6 months. Stroke. 1999 Apr; 30(4):744-8.

4 Kalf JG, de Wit S. Orofaryngeale slikstoornissen bij neurodegeneratieve aandoeningen. Tijdschr Gerontol Geriatr. 2014 Oct;45(5):27381.
5 Bakheit AM. Management of neurogenic dysphagia. Postgrad Med J. 2001 Nov;77(913): 694-9.

6 Ekberg O, Hamdy S, Woisard V, WuttgeHannig A, Ortega P. Social and psychological burden of dysphagia: its impact on diagnosis and treatment. Dysphagia. 2002;17(2):13946.
48

Folia Phoniatr Logop 2021;73:42-49 DOI: $10.1159 / 000504222$
Samuels et al. 
7 Florie M, Baijens L, Kremer B, Kross K, Lacko $\mathrm{M}$, Verhees $\mathrm{F}$, et al. Relationship between swallow-specific quality of life and fiber-optic endoscopic evaluation of swallowing findings in patients with head and neck cancer. Head Neck. 2016 Apr;38(S1 Suppl 1):E1848-56.

8 Pilz W, Baijens LW, Passos VL, Verdonschot $\mathrm{R}$, Wesseling F, Roodenburg N, et al. Swallowing assessment in myotonic dystrophy type 1 using fiberoptic endoscopic evaluation of swallowing (FEES). Neuromuscul Disord. 2014 Dec;24(12):1054-62.

9 McHorney CA, Martin-Harris B, Robbins J, Rosenbek J. Clinical validity of the SWALQOL and SWAL-CARE outcome tools with respect to bolus flow measures. Dysphagia. 2006 Jul;21(3):141-8.

10 McHorney CA, Robbins J, Lomax K, Rosenbek JC, Chignell K, Kramer AE, et al. The SWAL-QOL and SWAL-CARE outcomes tool for oropharyngeal dysphagia in adults: III. Documentation of reliability and validity. Dysphagia. 2002;17(2):97-114.

11 Bogaardt HC, Speyer R, Baijens LW, Fokkens WJ. Cross-cultural adaptation and validation of the Dutch version of SWAL-QoL. Dysphagia. 2009 Mar;24(1):66-70.

12 Vanderwegen J, Van Nuffelen G, De Bodt M The validation and psychometric properties of the Dutch version of the Swallowing Quality-of-Life Questionnaire (DSWAL-QOL). Dysphagia. 2013 Mar;28(1):11-23.

13 Woisard V, Andrieux MP, Puech M. [Validation of a self-assessment questionnaire for swallowing disorders (Deglutition Handicap Index)]. Rev Laryngol Otol Rhinol (Bord). 2006;127(5):315-25.

14 Speyer R, Heijnen BJ, Baijens LW, Vrijenhoef FH, Otters EF, Roodenburg N, et al. Quality of life in oncological patients with oropharyngeal dysphagia: validity and reliability of the
Dutch version of the MD Anderson Dysphagia Inventory and the Deglutition Handicap Index. Dysphagia. 2011 Dec;26(4):407-14.

15 Silbergleit AK, Schultz L, Jacobson BH, Beardsley T, Johnson AF. The Dysphagia handicap index: development and validation. Dysphagia. 2012 Mar;27(1):46-52.

16 Guns C, Van den Steen L, Benoy A, Van Nuffelen G, Verhaeghe E, Aerts A, et al. Vertaling en validering dysfagie handicap index. Logopedie. 2014;27:71-83.

17 Chen AY, Frankowski R, Bishop-Leone J, Hebert T, Leyk S, Lewin J, et al. The development and validation of a dysphagia-specific qualityof-life questionnaire for patients with head and neck cancer: the M. D. Anderson dysphagia inventory. Arch Otolaryngol Head Neck Surg. 2001 Jul;127(7):870-6.

18 Baijens LW. Multidisciplinaire polikliniek voor dysfagie. NtvKNO. 2011 Jan;18(1):5-6.

19 Folstein MF, Folstein SE, McHugh PR. "Minimental state". A practical method for grading the cognitive state of patients for the clinician. J Psychiatr Res. 1975 Nov;12(3):189-98.

20 de Campos RJ, Palma PV, Leite IC. Quality of life in patients with dysphagia after radiation and chemotherapy treatment for head and neck tumors. J Clin Exp Dent. 2013 Jul; 5(3):e122-7.

21 Finizia C, Rudberg I, Bergqvist H, Rydén A. A cross-sectional validation study of the Swedish version of SWAL-QOL. Dysphagia. 2012 Sep;27(3):325-35.

22 Terwee CB, Bot SD, de Boer MR, van der Windt DA, Knol DL, Dekker J, et al. Quality criteria were proposed for measurement properties of health status questionnaires. J Clin Epidemiol. 2007 Jan;60(1):34-42.

23 Evans JD. Straightforward statistics for the behavioral sciences. California: Brooks/Cole Publishing; 1996.
24 Carlsson S, Rydén A, Rudberg I, Bove M, Bergquist $H$, Finizia C. Validation of the Swedish M. D. Anderson Dysphagia Inventory (MDADI) in patients with head and neck cancer and neurologic swallowing disturbances. Dysphagia. 2012 Sep;27(3):361-9.

25 Guedes RL, Angelis EC, Chen AY, Kowalski LP, Vartanian JG. Validation and application of the M.D. Anderson Dysphagia Inventory in patients treated for head and neck cancer in Brazil. Dysphagia. 2013 Mar;28(1):24-32.

26 Kwon CH, Kim YH, Park JH, Oh BM, Han TR. Validity and reliability of the korean version of the MD anderson Dysphagia inventory for head and neck cancer patients. Ann Rehabil Med. 2013 Aug;37(4):479-87.

27 Matsuda Y, Kanazawa M, Komagamine Y, Yamashiro M, Akifusa S, Minakuchi S. Reliability and Validity of the MD Anderson Dysphagia Inventory Among Japanese Patients. Dysphagia. 2018 Feb;33(1):123-32.

28 Schindler A, Borghi E, Tiddia C, Ginocchio D, Felisati G, Ottaviani F. Adaptation and validation of the Italian MD Anderson dysphagia inventory (MDADI). Rev Laryngol Otol Rhinol (Bord). 2008;129(2):97-100.

29 Bauer F, Seiss M, Grässel E, Stelzle F, Klotz M, Rosanowski F. [Swallowing-related quality of life in oral cavity cancer. The German version of the Anderson Dysphagia Inventory]. HNO. 2010 Jul;58(7):692-7.

30 Zhang LJ, Jiang N, Li Z, Chen XW, Wang PG, Wang X, et al. Psychometric Properties of the Chinese Version of the M.D. Anderson Dysphagia Inventory for Head and Neck Cancer Patients. Cancer Nurs. 2017 May/Jun; 40(3):E9-16

31 Higginson IJ, Carr AJ. Measuring quality of life: using quality of life measures in the clinical setting. BMJ. 2001 May;322(7297):1297300 